\title{
GMR
}

\section{Cloning and characterization of defense-related genes from Populus szechuanica infected with rust fungus Melampsora larici-populina}

\author{
Z.J. Chen ${ }^{1,2}$, Z.M. Cao', Z.D. $\mathrm{Yu}^{1}$ and D. $\mathrm{Yu}^{1}$ \\ ${ }^{1}$ College of Forestry, Northwest A\&F University, Yangling, Shaanxi, China \\ ${ }^{2}$ College of Forestry and Landscape Architecture, \\ South China Agricultural University, Guangzhou, Guangdong, China \\ Corresponding author: Z.M. Cao \\ E-mail: zmcao@nwsuaf.edu.cn \\ Genet. Mol. Res. 15 (1): gmr.15017314 \\ Received July 28, 2015 \\ Accepted October 21, 2015 \\ Published February 19, 2016 \\ DOI http://dx.doi.org/10.4238/gmr.15017314
}

\begin{abstract}
Characterization of defense-related genes is critical for breeding disease-resistant poplar varieties and for better management and control of leaf rust disease. In the present study, full-length cDNAs of five Populus szechuanica defense-related (PsDR) genes, pathogen-related protein 1 (PsPR1), $\beta-1,3-g l u c a n a s e ~(P s G n s)$, thaumatin-like protein 1 (PsTLP1), thaumatin-like protein 2 (PsTLP2), and phenylalanine ammonialyase (PSPAL), were cloned from the leaves of $P$. szechuanica infected with Melampsora larici-populina (MLP). PsPR1 (728 bp), PsGns (1189 bp), PsTLP1 (929 bp), PSTLP2 (885 bp), and PSPAL (2586 bp) were predicted to encode $161,347,245,225$, and 711 amino acid residue-containing proteins with isoelectric points of $8.53,4.96,4.51,7.32$, and 5.87 , respectively. Moreover, the deduced PsDR proteins displayed more than $90 \%$ similarity to proteins from other Populus species. In response to the avirulent isolate, $\mathrm{Sb}_{052}$, and the virulent isolate, $\mathrm{Th}_{053}$, of MLP, the expression of PSDR genes was rapidly up-regulated in the leaves of $P$. szechuanica, peaked at 2 or 7 days post-inoculation (dpi), with levels in the incompatible interaction being higher than those in the compatible interaction. Meanwhile, the expression of
\end{abstract}


PsDR genes (except for PsGns) was also differentially up-regulated at 3, 7, or $18 \mathrm{dpi}$ in the petioles of the infected leaves, leaves next to the inoculated leaves, and in the top buds of the infected plants, respectively, compared to that at $0 \mathrm{dpi}$. These results suggest that these PsDR genes could play distinctive roles in the defense response of poplar against rust infection.

Key words: Poplar; Leaf rust; Defense response; Pathogenesis-related gene; Gene expression pattern

\section{INTRODUCTION}

Poplars are extensively cultivated worldwide. During their lifetime, they encounter various biotic stresses imposed by pathogenic fungi, bacteria, viruses, and insects, resulting in unhealthy growth, epidemics, and even death. Poplars have therefore developed diverse and multi-layered defense systems to govern tree health and limit damage (Duplessis et al., 2009). These physiological and biochemical defense systems include cell wall thickening, deposition of lignin as a physical barrier, generation of reactive oxygen species, and synthesis of anti-microbial proteins and a wide range of secondary metabolites, such as phenolic derivatives, upon pathogen attack (Rinaldi et al., 2007; Chen et al., 2010). Pathogenesis-related (PR) proteins play a critical role in plant defense against pest and pathogen attack, as well as, abiotic stress; expression of genes encoding PR-1, PR-2, PR-3, PR-5, PR-6, PR-9, PR-10, PR-12, and PR-14 has been reported in tree species on pathogen infection and abiotic stress (Veluthakkal and Dasgupta, 2010). PR-1 is believed to be involved in plant defense through a yet unknown mechanism, PR-1 genes have been reported to be differently regulated in different Populus-Melampsora interactions (Miranda et al., 2007; Rinaldi et al., 2007). The PR-2 family consists of $\beta-1$,3-glucanases, which directly hydrolyze $\beta-1,3$-glucan in the fungal cell wall, generating oligosaccharide elicitors that enhance host defense response (Klarzynski et al., 2000). Thaumatinlike proteins (TLP) are classified as members of the PR-5 family, widely distributed in animals, fungi, and plants and extensively studied in plants for their antifungal property (Liu et al., 2010; Wang et al., 2010; Petre et al., 2011). Phenylalanine ammonia-lyase (PAL), a key enzyme in the phenylpropanoid pathway, plays an important regulatory role in the biosynthesis of all phenylpropanoid products, such as flavonoids, phenylpropanoids, and lignin in plants (Bagal et al., 2012).

Poplar leaf rust, one of the most serious diseases that affect poplars, caused by the biotrophic rust fungus Melampsora larici-populina (MLP), leads to dramatic economic and ecological losses in poplar plantations, worldwide (Covarelli et al., 2013; Wan et al., 2013). During the last decades, breeding resistant poplar was considered an effective and environment-friendly approach to control this disease or to alleviate damage caused by it. However, culture practices of monoclonal plantations and directional selection of rust fungus enhanced the rapid breakdown of selected poplar resistances (Pinon and Frey, 2005). It is, thus, crucial to develop new strategies for controlling this disease. This especially requires a better understanding of the recognition system and the host self-defense mechanisms in the Populus-Melampsora pathosystem (Duplessis et al., 2009; Hacquard et al., 2011). In this study, five Populus szechuanica defense-related $(P s D R)$ genes were cloned from the leaves of $P$. szechuanica, a widely distributed species of poplar in China, which is resistant to most strains of MLP. Additionally, the spatio-temporal expression patterns of these PsDR genes were detected in $P$. szechuanica during the infection process of MLP. 


\section{MATERIAL AND METHODS}

\section{Treatment and sample materials}

P. szechuanica plants (2-3 years old) were collected from the Qinling Mountain of China. The plant cuttings were then potted and grown in a greenhouse for 12 weeks at $\sim 20^{\circ}-26^{\circ} \mathrm{C}$. The MLP urediniospores were multiplicated, collected, dried, and stored as reported earlier (Chen et al., 2014). MLP isolates $\mathrm{Sb}_{052}$ (avirulent) and $\mathrm{Th}_{053}$ (virulent) were inoculated on the leaves of $P$. szechuanica, which resulted in incompatible and compatible interactions, respectively, between the poplar and the rust fungus. Fully expanded leaves with a leaf plastochron index of 5 to 8 were daubed on their abaxial surface with urediospore suspensions $(1-2 \mathrm{mg} / \mathrm{mL})$ of $\mathrm{Sb}_{052}$ or $\mathrm{Th}_{053}$ isolates, and the leaves next to the inoculated leaves $(\mathrm{NL})$ were sprayed with tap water. The inoculated plants were placed in a growth chamber with high humidity for $24 \mathrm{~h}$ and were subsequently cultured in greenhouse with natural light at $\sim 25^{\circ}-27^{\circ} \mathrm{C}$. To detect the change in $P s D R$ expression in different organs of $P$. szechuanica after infection with $\mathrm{Sb}_{052}$, the petioles (SP), roots (SR), top buds (SB), and the NL were harvested at 0 (used as the control), 3, 7, and 18 days post-inoculation (dpi). To further understand the PSDR expression pattern in the leaves of poplar during the interactions with two MLP isolates, samples of the leaves inoculated with $\mathrm{Sb}_{052}(\mathrm{SL})$ or $\mathrm{Th}_{053}(\mathrm{TL})$ and the leaves next to the SL (SNL) or TL (TNL) were harvested at 0 (used as the control), 0.5, 1, 2, 3, 4, 7, and $18 \mathrm{dpi}$. Samples collected at each time point from three individual plants were pooled, immediately snapfrozen in liquid nitrogen, and stored at $-80^{\circ} \mathrm{C}$ until used for total RNA extraction.

\section{Total RNA extraction and cDNA synthesis}

Total RNA was extracted from 100-mg samples using the UNIQ-10 Column TRIzol Total RNA Extraction Kit following the manufacturer instructions (Sangon Biotech, Shanghai, China). In order to minimize the variations between individual RNA samples, samples of each organ from three individual plants collected at respective time points were pooled. The quantity and quality of the total RNA was assessed using the NanoDrop ND-1000 spectrophotometer (Thermo Fisher Scientific, Wilmington, DE, USA) and gel electrophoresis, respectively. For amplification of the 5'and 3'-end fragments of PsDR, equal amounts of total RNA from SL, collected at each time point, were pooled together and translated into adaptor-ligated cDNA using the SMARTer ${ }^{\text {TM }}$-RACE cDNA Amplification Kit (Clontech, Palo Alto, CA, USA) following the manufacturer recommendations. For the real time-quantitative polymerase chain reaction (RT-qPCR) detection of PsDR expression patterns, $1 \mathrm{mg}$ total RNA from each sample was used to synthesize cDNA by the PrimeScript ${ }^{\circledR}$ RT Reagent Kit with gDNA Eraser (TaKaRa, Dalian, China) according to the manufacturer instructions.

\section{Rapid amplification of cDNA ends}

To clone the P. szechuanica pathogen-related protein 1 (PsPR1), $\beta$-1,3-glucanase (PsGns), thaumatin-like protein 1 (PsTLP1), thaumatin-like protein 2 (PSTLP2), and phenylalanine ammonialyase (PSPAL), specific primers (shown in Table 1) for rapid amplification of 5'- and 3'-end fragments were designed, based on the sequences in the suppression subtractive hybridization-cDNA library of $P$. szechuanica infected with MLP, using the premier PRIMER v5.0 software (PREMIER Biosoft International, Palo Alto, CA, USA) (Chen et al., 2014). Nested PCR was performed on a PTC- 
200 Programmable Thermal Cycler (MJ Research Inc., Waltham, MA, USA) using TaKaRa Taq ${ }^{\mathrm{TM}}$ Hot Start polymerase (TaKaRa); a 50- $\mu \mathrm{L}$ reaction contained $2 \mu \mathrm{L}$ adaptor-ligated cDNA (in the first-round) or the first-round PCR products diluted 50 times (in the second-round) as template, $5 \mu \mathrm{L}$ Universal Primer A Mix (in the first-round) or nested universal primer (in the second-round) (supplied in the TaKaRa Taq ${ }^{\mathrm{TM}}$ Hot Start polymerase kit), $1 \mu \mathrm{L}$ gene specific primer 1 (GSP1, $10 \mu \mathrm{M}$, for the first-round) or gene specific primer 2 (GSP2, $10 \mu \mathrm{M}$, for the second-round), $5 \mu \mathrm{L} 10 \mathrm{X}$ PCR buffer, $8 \mu \mathrm{L}$ dNTP mixture, and 2.5 U TaKaRa Taq Hot Start version (TaKaRa). The PCR conditions for the rapid amplification of cDNA ends (RACE) were as follows: 5 cycles at $94^{\circ} \mathrm{C}$ for $30 \mathrm{~s}, 70^{\circ} \mathrm{C}$ for $30 \mathrm{~s}, 72^{\circ} \mathrm{C}$ for $1 \mathrm{~min}, 5$ cycles at $94^{\circ} \mathrm{C}$ for $30 \mathrm{~s}, 70^{\circ} \mathrm{C}$ for $30 \mathrm{~s}, 72^{\circ} \mathrm{C}$ for $1 \mathrm{~min}, 25$ cycles at $94^{\circ} \mathrm{C}$ for $30 \mathrm{~s}, 68^{\circ} \mathrm{C}$ for $30 \mathrm{~s}$, and $72^{\circ} \mathrm{C}$ for $1 \mathrm{~min}$, and a final extension at $72^{\circ} \mathrm{C}$ for $10 \mathrm{~min}$. The second-round PCR products were separated on a 1.2\% agarose gel and purified using the TaKaRa MiniBEST Agarose Gel DNA Extraction Kit (TaKaRa). The PCR products were cloned in a pGEM-T easy vector system (TaKaRa) and transformed into Escherichia coli DH5 $\alpha$ competent cells according to the manufacturer instructions. The positive clones were identified by colony PCR and sequenced using M13 forward and reverse primers by Sangon Biotech Company.

\begin{tabular}{|c|c|c|c|c|}
\hline Gene & Primer & Sequence (5' to $\left.3^{\prime}\right)$ & Size (bp) & $\operatorname{Tm}\left({ }^{\circ} \mathrm{C}\right)$ \\
\hline \multirow{4}{*}{ PsPR1 } & 5'-GSP1 & CCACTACTCGCTGCAAGGTTCTCGCCA & 27 & 68.1 \\
\hline & 5'-GSP2 & TCTGCAATCGCCCGTGAGCCGTTTAAT & 27 & 65 \\
\hline & 3'-GSP1 & СССТСТАТСССТTGСССАAAACTCCCC & 27 & 68.1 \\
\hline & 3'-GSP2 & CGGCTCACGGGCGATTGCAGACTTGT & 26 & 68.3 \\
\hline \multirow{4}{*}{ PsGns } & 5'-GSP1 & GTTCAAATCTGGGGCACCTGCTTTCTCG & 28 & 66.4 \\
\hline & 5'-GSP2 & TCCGACGGAGGGTAGGAGCTGCCCAACA & 28 & 70.8 \\
\hline & 3'-GSP1 & CCGTCGGATGGGTCCTTTAGTGACAGTG & 28 & 67.9 \\
\hline & 3'-GSP2 & CGAGAAAGCAGGTGCCCCAGATTTGAAC & 28 & 66.4 \\
\hline \multirow{4}{*}{ PSTLP1 } & 5'-GSP1 & CGATCCTTTTGGGATATGTCAATCCC & 26 & 62 \\
\hline & 5'-GSP2 & GCAATCACAGTCCCATCTGAACCTCTC & 27 & 65 \\
\hline & 3'-GSP1 & GCTATAATTACCTTCACCCTCGTCATC & 27 & 62 \\
\hline & 3'-GSP2 & GCTGGCGGTCCGTCTTTATCT & 25 & 62.9 \\
\hline \multirow{4}{*}{ PSTLP2 } & 5'-GSP1 & TATTCAGCCAGTGTGTTAGGGGGT & 24 & 62 \\
\hline & 5'-GSP2 & GTGTGTTAGGGGGTTGCCCGAAGG & 24 & 67.1 \\
\hline & 3'-GSP1 & CAAGCCTTCGGGCAACCCCCTAACA & 25 & 66.9 \\
\hline & 3'-GSP2 & CAACCCCCTAACACACTGGCTGAA & 24 & 63.7 \\
\hline \multirow{4}{*}{$P s P A L$} & 5'-GSP1 & CCCCCATTTTGGCATAGAGGATCATCG & 27 & 65 \\
\hline & 5'-GSP2 & ATGGCGGCAGAGTCACTAAAAGGAAGC & 27 & 65 \\
\hline & 3'-GSP1 & GTGAACCATTGCCATTGCGTGAATCTTG & 28 & 63.5 \\
\hline & 3'-GSP2 & GCCAAAATGGGGGAGAGACAAAAAAAGA & 28 & 62 \\
\hline
\end{tabular}

\section{Sequence comparisons and phylogenetic analysis}

The vector sequence was removed using the VecScreen tool (http://www.ncbi.nlm.nih. gov/tools/vecscreen/). The full-length sequence of each PsDR was obtained by assembling its 5'- and 3'-end sequences, and was submitted to GenBank. The nucleotide and predicted protein sequences were searched against the plant nucleotide and protein database using BLASTn and BLASTp algorithms (http://www.ncbi.nlm.nih.gov), respectively. The open reading frame was predicted using the ORF Finder (http://www.ncbi.nlm.nih.gov/gorf/gorf.html). The deduced protein sequences were further analyzed using InterProScan (http://www.ebi.ac.uk/InterProScan/) and PROSITE (http://au.expasy.org/prosite/) softwares. The physical and chemical parameters were computed using the ProtParam tool (http://web.expasy.org/protparam/) and the signal peptide and transmembrane domain were predicted using the Signal P 4.1 Server (http://www.cbs.dtu. 
dk/services/SignalP/) and the TMHMM Server v. 2.0 (http://www.cbs.dtu.dk/services/TMHMM/), respectively. Subsequently, BLASTx was performed against the non-redundant NCBI database, and the secondary structure was analyzed using the SOPMA program (https://npsa-prabi.ibcp. fr/cgi-bin/npsa_automat.pl?page=/NPSA/npsa_sopma.html). Multiple alignments of the deduced proteins encoded by the PsDRs with proteins from other plants were performed using the ClustalW software. Phylogenetic trees were constructed using the Molecular Evolutionary Genetics Analysis (MEGA) package 5.0 program, using the neighbor-joining method with 1000 replicates bootstrap analysis to achieve the confidence levels required for the phylogenetic analysis.

\section{Spatio-temporal expression patterns of PsDRs in P. szechuanica infected with MLP}

For amplification of specific transcripts by RT-qPCR, specific primers were designed using the premier PRIMER v5.0 software (PREMIER Biosoft International) based on the fulllength sequence of the PsDRs (detailed in Table 2). The Populus ubiquitin (Rinaldi et al., 2007) gene was used as an endogenous reference to normalize the amount of total RNA used in each reaction. The RT-qPCRs were performed in an iQ5 ${ }^{T M}$ Real-time PCR system (Bio-Rad, Hercules, $\mathrm{CA}$, USA); the $25-\mu \mathrm{L}$ mixture contained $2 \mu \mathrm{L} \mathrm{cDNA}$ (five times diluted), $1 \mu \mathrm{M}$ specific primers for the $P s D R$ or reference gene and the reaction was carried out following the SYBR Green PCR Master Mix Kit manufacturer instructions (TaKaRa). The levels of $P s D R s$ in the organ samples from the different time points were compared to those observed on $0 \mathrm{dpi}$, using the Delta-Delta Comparative Threshold method (Livak and Schmittgen, 2001), to determine the relative expression.

Table 2. Primers used in RT-qPCR for expression analysis of PsDR genes.

\begin{tabular}{l|l|l|r}
\hline Gene & Forward (5' primer) & Reverse (3' primer) & Amplicon length (bp) \\
\hline PsPR1 & CATGTGTTGGTGGAGAATGC & TCGCGGAAATACATCGTTTC & 253 \\
\hline PsTLP1 & AGCACCCGTTGCCAATGTGAAGCCAGTT & GCTATAATTACCTTCACCCTCGTCATC & 159 \\
\hline PsTLP2 & GGAGAGTCGTATGCCCATTGCTA & CAGTTGAAGATAAAGGCGGACCG & 179 \\
\hline PsGns & CCGTCGGATGGGTCCTTTAGTGACAGTG & GTTCAAATCTGGGGCACCTGCTTTCTCG & 270 \\
\hline PsPAL & GCAACCTCACATAGAAGAACCAAGC & AGACTCCGTGCCATTCCCAAAGATA & 96 \\
\hline Ubiquitin & GCAGGGAAACAGTGAGGAAGG & TGGACTCACGAGGACAG & 151 \\
\hline
\end{tabular}

\section{RESULTS}

\section{Cloning of five PsDR genes}

The full-length sequences of PSPR1, PsGns, PsTLP1, PsTLP2, and PSPAL were cloned from the leaves of $P$. szechuanica infected with MLP. The 5'-RACE and 3'-RACE of the five PsDRs was performed using the adaptor-ligated cDNA, which was reverse-transcribed from the total RNA extracted from the MLP infected P. szechuanica leaves (SL) at 1, 2, 3, 4, and 7 dpi (Figure 1). Each full-length cDNA sequence (GenBank accession Nos. KP109917, KP109918, KP109919, KP109920, and KP109921) was obtained by assembling the sequences of the 5'- and 3'- cDNA end fragments.

\section{Sequence analysis of $P s D R$ genes}

Nucleotide sequence of each PsDR consisted of an untranslated region (UTR), a poly(A) tail, a eukaryotic initiator codon (ATG), and the typical polyadenylation signal sequence (AATAAA) 
at the 3'-UTR. The full-length cDNAs of the PSPR1, PsGns, PsTLP1, PsTLP2, and PSPAL were $728,1189,929,885$, and 2586 bp long, including full-length open reading frames encoding 161 , $347,245,225$, and 711 amino acids (aa), respectively (Table 3 ). The predicted molecular weights of the deduced proteins encoded by PSPR1, PsGns, PsTLP1, PsTLP2, and PSPAL were 25.4, 24.8, $17.4,77.7$, and $37.4 \mathrm{kDa}$, with the theoretical isoelectric point (pl) at $8.53,4.96,4.51,7.32$, and 5.87 , respectively (Table 3). SignalP was identified in all the deduced PsDR proteins except for the PsPAL; the cleavage site was between amino acid position (pos.) 25 and 26, 34 and 35, 23 and 24, 25 and 26 in PsPR1, PsGns, PsTLP1, and PsTLP2, respectively. A transmembrane domain was predicted in the deduced PsPR1, PsGns, and PsTLP1 but not in PsTLP2 and PsPAL, indicating that PsTLP2 and PsPAL might be extracellular secreted proteins. Several phosphorylation sites were predicted in the deduced PsDRs. The observed secondary structures are shown in Table 4.
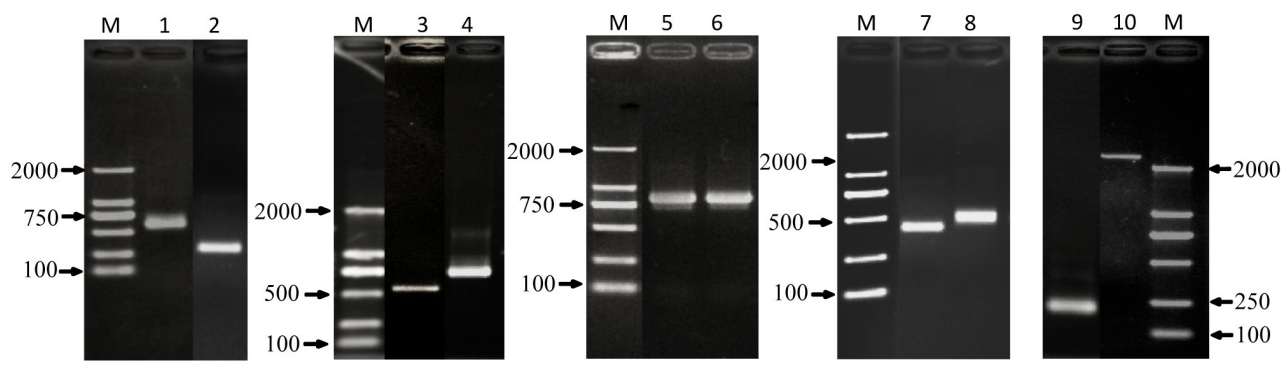

Figure 1. PCR amplification products of the 3'- and 5'-end fragments. Lane M: marker 2000, Lanes 1, 3, 5, 7, and $9=$ PCR amplification product of the 3 '-end fragment for the PSPR1, PsGns, PsTLP1, PsTLP2, PsPAL genes, respectively. Lanes 2, 4, 6, 8, and 10 = PCR amplification product of the 5'-end fragment for the PsPR1, PsGns, PsTLP1, PsTLP2, PSPAL genes, respectively.

Table 3. Characteristics of the PSDR genes in response to infection with Melampsora larici-populina.

\begin{tabular}{l|c|c|c|c|c}
\hline Gene & PSPR1 & PSGns & PSTLP1 & PSTLP2 & PSPAL \\
\hline GenBank accession No. & KP109919 & KP109921 & KP109917 & KP109918 & KP109920 \\
\hline Full length & 728 & 1189 & 929 & 885 & 2586 \\
\hline Number of amino acid & 161 & 347 & 245 & 225 & 711 \\
\hline Isoelectric point & 8.53 & 4.96 & 4.51 & 7.32 & 5.87 \\
\hline Molecular mass (kDa) & 17391.5 & 37442.9 & 25363.4 & 24753.7 & 77743 \\
\hline Formula & $\mathrm{C}_{759} \mathrm{H}_{1184} \mathrm{~N}_{220} \mathrm{O}_{236} \mathrm{~S}_{7}$ & $\mathrm{C}_{1666} \mathrm{H}_{2587} \mathrm{~N}_{445} \mathrm{O}_{523} \mathrm{~S}_{7}$ & $\mathrm{C}_{1110} \mathrm{H}_{1689} \mathrm{~N}_{285} \mathrm{O}_{356} \mathrm{~S}_{19}$ & $\mathrm{C}_{1082} \mathrm{H}_{1614} \mathrm{~N}_{304} \mathrm{O}_{327} \mathrm{~S}_{20}$ & $\mathrm{C}_{3429} \mathrm{H}_{5493} \mathrm{~N}_{949} \mathrm{O}_{1052} \mathrm{~S}_{28}$ \\
\hline Grand average of hydropathicity & -0.257 & -0.093 & -0.100 & -0.291 \\
\hline
\end{tabular}

Table 4. Predicted phosphorylation sites and the secondary structures of deduced proteins encoded by PsDR genes.

\begin{tabular}{l|c|c|c|c|c|c|c}
\hline Gene & Ser & Thr & Tyr & Alpha helix & Extended strand & Beta turn & Random coil \\
\hline PsTLP1 & 9 & 3 & 2 & $10.61 \%$ & $24.90 \%$ & $4.90 \%$ & $59.59 \%$ \\
\hline PSTLP2 & 3 & 2 & 3 & $7.56 \%$ & $26.67 \%$ & $3.11 \%$ & $62.67 \%$ \\
\hline PSPR1 & 5 & 2 & 8 & $22.98 \%$ & $28.57 \%$ & $11.18 \%$ & $37.27 \%$ \\
\hline PSPAL & 24 & 8 & 7 & $50.07 \%$ & $12.80 \%$ & $9.14 \%$ & $27.99 \%$ \\
\hline PSGns & 14 & 4 & 2 & $42.07 \%$ & $20.75 \%$ & $9.80 \%$ & $27.38 \%$ \\
\hline
\end{tabular}

\section{Structural characterization of deduced proteins encoded by the PsDR genes}

The deduced protein encoded by PsPR1 belonged to the sterol carrier protein (SCP) superfamily, and contained the SCP_PR-1_like domain and the domains of cysteine-rich secretory 
proteins antigen 5 and PR-1 (CAP). It contained six highly conserved cysteine residues and a conserved C[R/G]HYTQVVWRxS[V/A]RxGC motif (van Loon and Van Strien, 1999; Figures 2

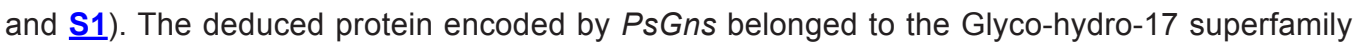
(Fiqure S1), whereas those encoded by PSTLP1 and PSTLP2 belonged to the typical PR-protein GH64-TLP-SF superfamily, members of which contain the conserved TLP-PA and THN domains and a thaumatin family signature (PS00316; G-x-[GF]-x-C-x-T-[GA]-D-C-x(1,2)-[GQ]-x(2,3)-C;

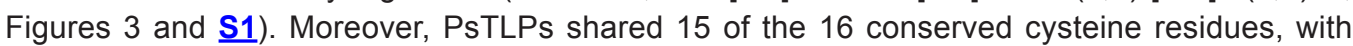
only one cysteine difference at the conserved positions between PsTLP1 (at pos. 150) and PsTLP2 (at pos. 141). The basic electronegative/electropositive cleft region and a conserved REDDD motif were identified in the PsTLPs, and a predicted IgE-binding epitope region was found in PsTLP1 (Figure 3). In addition, PSPAL belonged to the Lyase_I_like superfamily, which contains a phenylalanine/histidine ammonia-lyase (PAL-HAL) domain (active sites), a tetramer interface (polypeptide binding site), as well as a conserved PAL protein finger motif (GTITASGDLVPLSYIAG), which is a typical tag of PAL-HAL domain (Figures 4 and $\underline{\mathbf{S 1}}$ ).

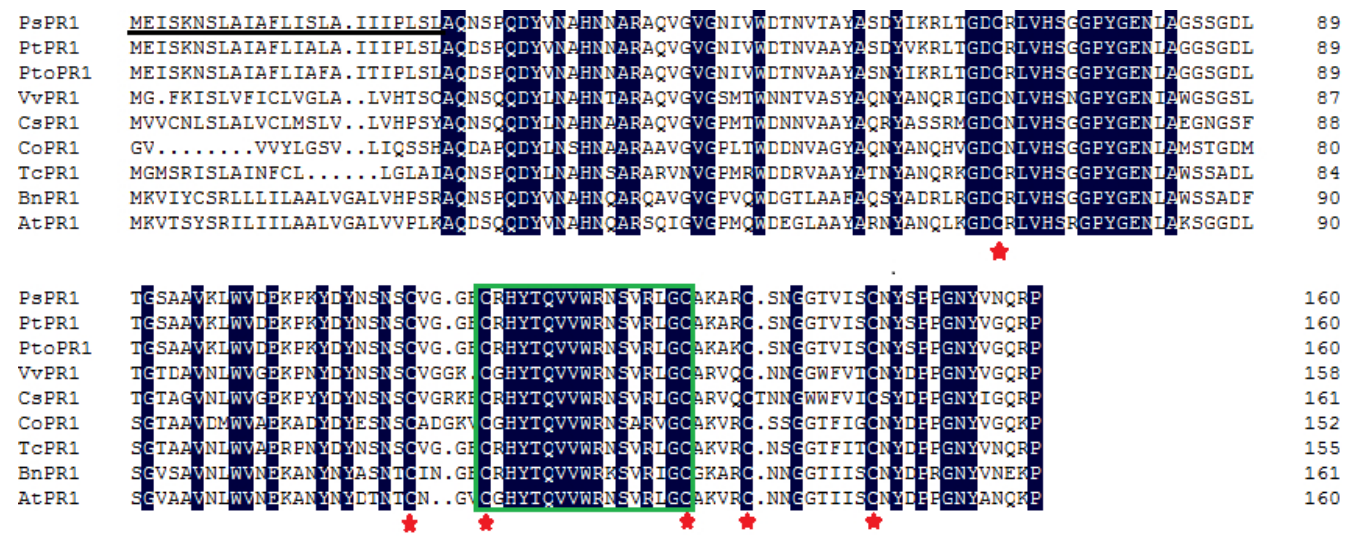

Figure 2. Multiple alignment and conserved domain of PsPR1 with PR1 from various plant species. Amino acid (aa) sequence comparison was carried out with ClustalW on MEGA 5 software with the parameters described by Shatters Jr. et al. (2006) and manually adjusted. The predicted signal peptide and conserved positions of aa are underlined and labeled with black, respectively. The conserved cysteine residue position is indicated by a star, and the PR1 family conserved motif C[R/G]HYTQVVWRxS[V/A]RxGC is boxed in green. The first two (or three in Populus) letters correspond to the initial letter(s) of the genus name and the species name of the organism. Sequence names and GenBank accession Nos. are as follows: Populus trichocarpa, PtPR1 (XP_006369711.1); Populus tomentosa, PtoPR1 (ADP69172.1); Theobroma cacao, TcPR1 (XP_007041422.1); Brassica napus, BnPR1 (AAB06458.1); Vitis vinifera, VvPR1 (ADN43427.1); Arabidopsis thaliana, AtPR1 (NP_179064.1); Cydonia oblonga, CoPR1 (AFK64734.1); Camellia sinensis, CsPR1 (AHA56682.1).

\section{BLASTp analysis of PsDRs and phylogenetic tree construction}

The five PsDRs showed more than 90\% identity with proteins from the Populus species. In the phylogenetic trees, the deduced proteins PsPR1, PsGns, and PsPAL were grouped with PR1, Gns, and PAL proteins, respectively, from Populus species as a clade, suggesting that PR1, Gns, and PAL proteins are highly conserved in Populus (Figures S2, $\underline{\text { S3}}$, and $\underline{\mathbf{S 4}}$ ). Moreover, PsPR1 showed $77 \%$ similarity to Theobroma cacao PR1, $70 \%$ similarity to Arabidopsis thaliana 


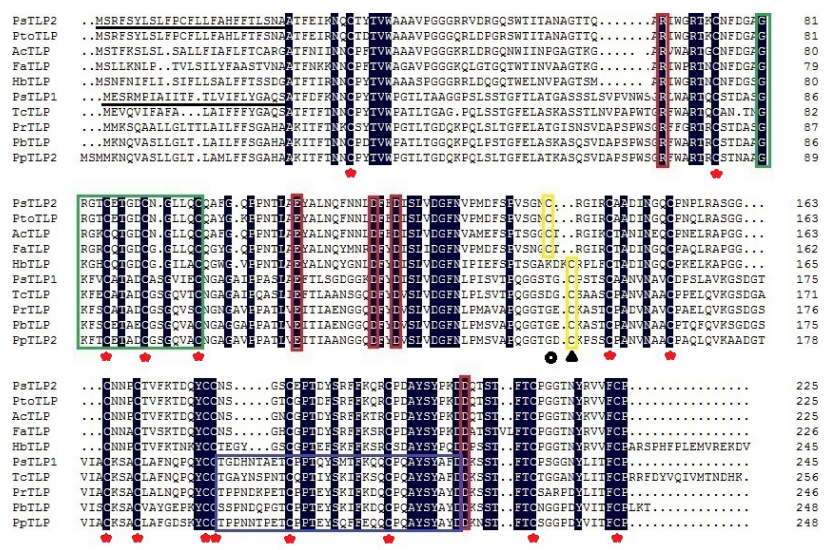

Figure 3. Multiple alignment and conserved domains of PsTLP with TLP from various plant species. Amino acid (aa) sequence comparison was carried out with ClustalW on MEGA 5 software with the parameters described by Shatters Jr. et al. (2006) and manually adjusted. The predicted signal peptide and conserved positions of aa sequence are underlined and labeled with black, respectively. The TLP family signature (PS00316) in thaumatin, G-x-[GF]-x-C-x-T[GA]-D-C-x(1,2)-[GQ]-x(2,3)-C is boxed in green. Fifteen conserved cysteine residue sites in both PsTLP1 and PsTLP2 are indicated by stars, and other conserved cysteine residue sites at 150 in PSTLP1 and at 141 in PSTLP2 are boxed in yellow and indicated by a circle and triangle, respectively. A REDDD motif and a predicted IgE-binding epitope domain are indicated by red and blue boxes, respectively. In addition, the first two (or three in Populus) letters correspond to the initial letter(s) of the organism genus and species names. Sequence name and GenBank accession Nos. are as follows: Populus tomentosa, PtoTLP (ADP69173.1); Actinidia chinensis, AcTLP (AGC39176.1); Fragaria $x$ ananassa, FaTLP (AAF13707.1); Hevea brasiliensis, HbTLP (AHA83528.1); Theobroma cacao, TcTLP (XP_007027490.1); Prunus persica, PrTLP (AEV57471.1); Pyrus $x$ bretschneideri, PbTLP (ACX32461.1); Pyrus pyrifolia, PpT̄LP (ACN97419.1).

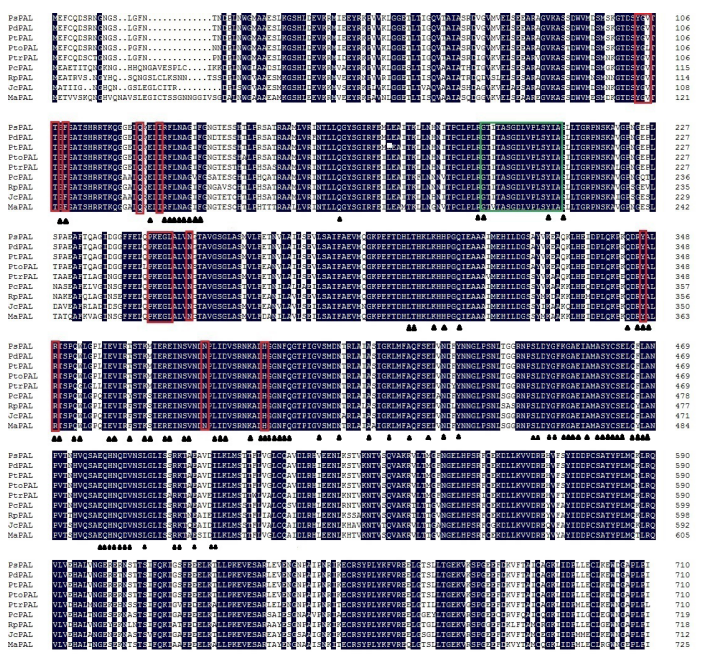

Figure 4. Multiple alignment and conserved domains of PsPAL with PAL from various plant species. Amino acid (aa) sequence comparison was carried out with ClustalW on MEGA 5 software with the parameters described by Shatters Jr. et al. (2006) and manually adjusted. The conserved PAL protein finger motif (GTITASGDLVPLSYIA) is boxed in green. The active site and the intramer interface (polypeptide binding site) are indicated by a red box and black triangle, respectively. The first two (or three in Populus) letters correspond to the initials of the organism genus and species names and sequence name and GenBank accession Nos. are as follows: Populus trichocarpa, PtPAL (XP_002315308.1); P. trichocarpa $\times$ P. deltoids, PdPAL (AAQ74878.1); P. tomentosa, PtoPAL (AFZ78653.1); P. tremuloides, PtrPAL (AF480620_1); Jatropha curcas, JcPAL (ABI33979.1); Morus alba, MaPAL (AID16077.1); Pyrus communis, PcPAL (AGL50914.1); Robinia pseudoacacia, RpPAL (ACF94716.1). 
and Vitis vinifera PR1, PsGns showed 69, 65, and 62\% similarity to Fragaria x ananassa, Pyrus pyrifolia, and Hevea brasiliensis Gns, respectively. PsPAL showed about $85 \%$ similarity to Pyrus communis, Robinia pseudoacacia, and Nicotiana attenuata PAL. PsTLP1 was grouped together with the proteins encoded by TcTLP, PrTLP, PbTLP, and PpTLP, and showed $71 \%$ similarity to T. cacao TLP1 and $63 \%$ to Pyrus pyrifolia and Prunus persica TLP1, whereas PsTLP2, clustered with PtoTLP, AcTLP, FaTLP, and HbTLP in another group (Figure 5) and showed 79, 77, and 69\% similarity to Actinidia chinensis, Fragaria $x$ ananassa and $H$. brasiliensis TLP2, respectively.

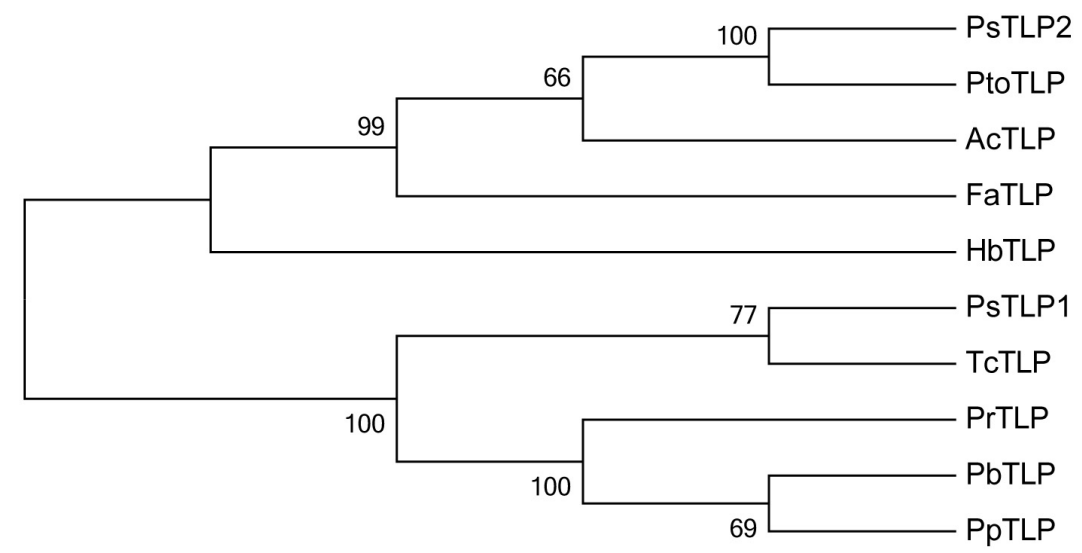

Figure 5. Phylogenetic tree of PsTLP with TLP from various plant species. The sequence name and GenBank accession Nos. are the same as in Figure 3.

\section{PsDR gene expression patterns in different organs of $P$. szechuanica induced by avirulent isolate of MLP}

In order to evaluate the behavior of $P S D R$ genes in the organs of poplar induced by rust fungi, the dynamics of the change in PsDR expression was determined in different organs of $P$. szechuanica inoculated with the avirulent isolate $\mathrm{Sb}_{052}$. In this incompatible interaction, the expression level of PSPAL and PSPR1 genes in the leaves and petioles was obviously upregulated $\sim 18-22$-fold and $\sim 20-25$-fold at 7 dpi compared with the levels on 0 dpi, respectively. PSPR1 expression increased by 24-fold and 7-fold at $3 \mathrm{dpi}$ in SP and SB, respectively, and then returned to the level of that at $0 \mathrm{dpi}$ in all organs (except SL). However, the PSPAL expression remained high in leaves (7-fold), petioles (15-fold), and NL (18-fold) at $18 \mathrm{dpi}$. The expression of PsGns and PSTLP1 was almost the same as that in uninduced state in SP, SB, and SR, but was dramatically up-regulated in SL at $7 \mathrm{dpi}$ with 294-fold and 88-fold increases, respectively. In addition, the expression of PSTLP2 was up-regulated 25-, 18-, 34-, 73-, and 24-fold at 3, 3, 7, 7, and $7 \mathrm{dpi}$, respectively, in all the organs (SL, NL, SP, SB, and SR; Figure 6) and was the highest.

On the whole, the PsDR expression levels were differentially increased at 3, 7, or $18 \mathrm{dpi}$ compared to that on $0 \mathrm{dpi}$ in the different organs of poplar infected with the rust fungus (Figure 6). Expression levels of PSPR1, PsTLP2, and PSPAL were dramatically up-regulated in both SP and SL. PSTLP2 and PSPAL were also dramatically up-regulated in SB and NL of infected plants at 7 and $18 \mathrm{dpi}$, respectively. However, the expression levels of PSPR1, PsGns, PsTLP1, and PSPAL in the SR seemed to be either unaffected or repressed by the MLP infection. 

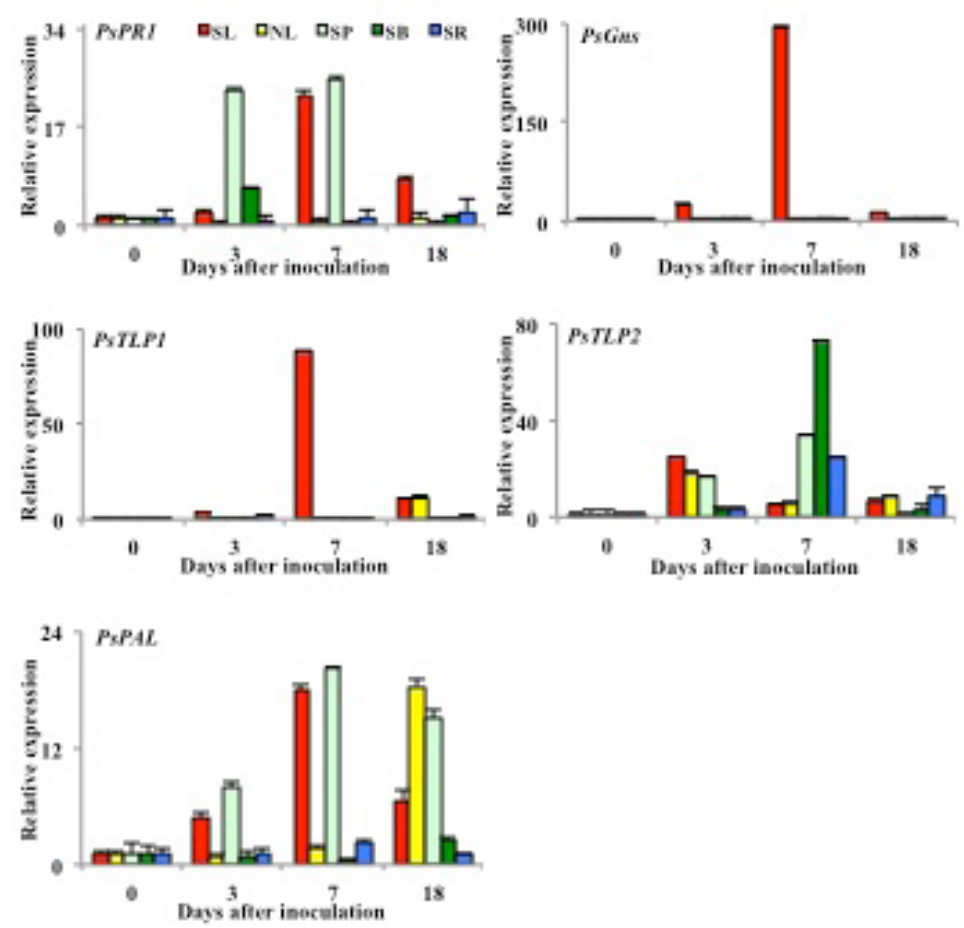

Figure 6. Relative expression of $P s D R$ genes in the organs of Populus szechuanica inoculated with the avirulent isolate $\mathrm{Sb}_{052}$ of Melampsora laricis-populina. The endogenous reference gene was Populus ubiquitin, and the normalized PsDR gene levels at 0 dpi was arbitrarily set to 1 . SL: leaves inoculated with MLP $\mathrm{Sb}_{052}$; $\mathrm{NL}$ : uninoculated leaves next to the inoculated leaves; SP: petioles of inoculated leaves; SB: top buds of inoculated plants; SR: roots of inoculated plants.

\section{PsDR gene expression patterns in poplar leaves of incompatible and compatible interaction between $P$. szechuanica and $M$. larici-populina}

Relative expression levels of the five $P S D R$ genes were further examined at more time points in the $P$. szechuanica leaves in response to the MLP isolates $\mathrm{Sb}_{052}$ and $\mathrm{Th}_{053}$. Expression levels of these genes were rapidly up-regulated at $0.5 \mathrm{dpi}$ in both the interactions. Expression levels of PSPR1 and PSTLP1 at $0.5 \mathrm{dpi}$ were higher in the incompatible interaction than the compatible interaction. The expression levels of PSPR1 and PSPAL peaked at $2 \mathrm{dpi}$, and were higher (38and 26-fold) in the incompatible interaction than in the compatible interaction (14- and 8-fold). However, the second expression of PsPR1 (at $4 \mathrm{dpi}$ ) in incompatible interaction peaked earlier than in the compatible interaction (at $7 \mathrm{dpi}$ ), whereas PSPAL showed a second peak of expression at $7 \mathrm{dpi}$ in both the interactions. The expression levels of PsGns and PSTLP1 also reached a second peak at $7 \mathrm{dpi}$ in the incompatible (294- and 88-fold) and the compatible (133- and 13-fold) interactions. The expression of PSTLP2 gene peaked earlier and was higher (at $4 \mathrm{dpi}$ with 35 -fold) in the incompatible interaction compared to that in the compatible interaction (at $7 \mathrm{dpi}$ with 22-fold). In addition, the expression patterns of PsDR genes in SNL and TNL were similar to that in the SL and TL, respectively, albeit with very low expression levels, except for PsTLP2 at 3 dpi (18-fold increase) in SNL (Figure 7). 

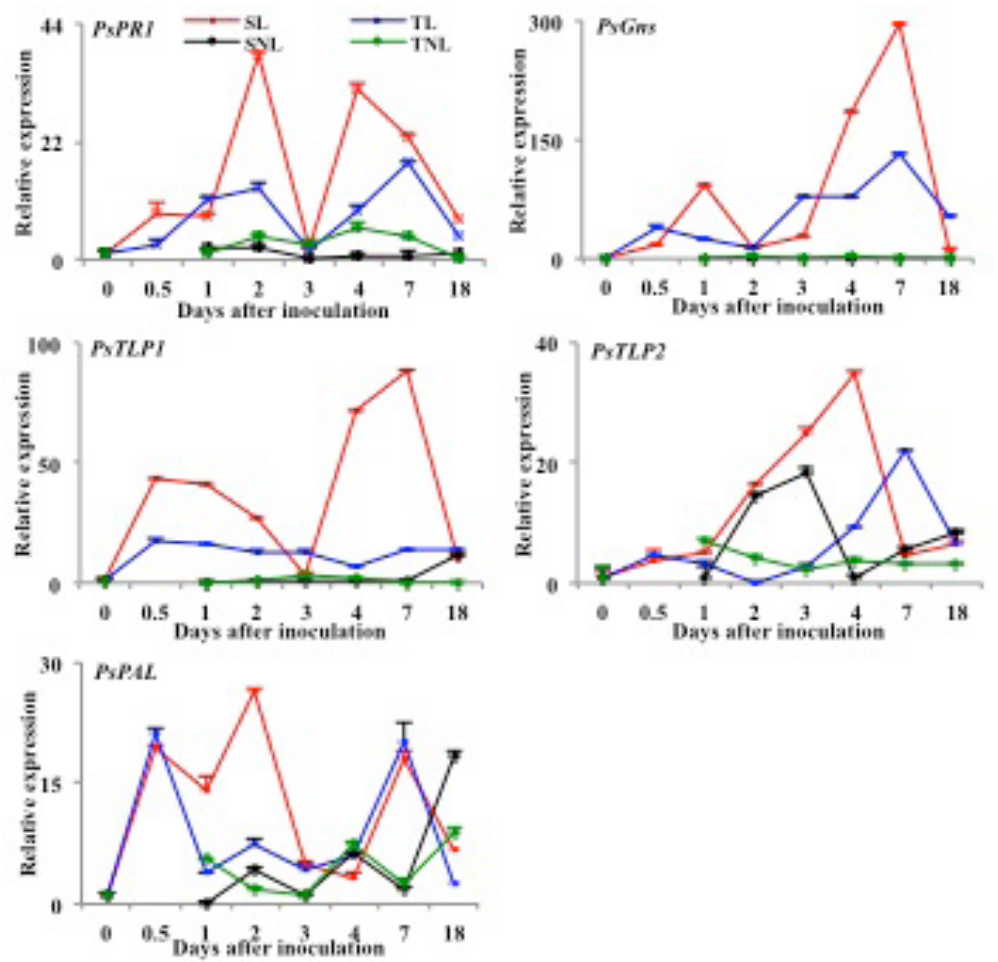

Figure 7. Temporal expession patterns of $P s D R$ genes during the infection time course of incompatible and compatible Populus-MLP interactions. The endogenous reference gene was Populus ubiquitin. The normalized $P s D R$ gene levels at 0 dpi were arbitrarily set to 1 . SL: leaves inoculated with $\mathrm{Sb}_{052}$ (incompatible interaction); TL: leaves inoculated with $\mathrm{Th}_{053}$ (compatible interaction); SNL: uninoculated leaves next to SL; TNL: uninoculated leaves next to TL.

\section{DISCUSSION}

In the present study, five PsDR genes were cloned and their spatio-temporal expression patterns in different organs of $P$. szechuanica, with or without infection with M. larici-populina, were analyzed. The deduced protein encoded by PSPR1 contained a signal sequence of 25 aa residues, which was similar to most of the PR1 (with a 24-26-aa signal sequence), and six conserved cysteine residues that usually form disulfide bonds (Fernández et al., 1997) were also found in PsPR1. PsPR1 with pl 8.5 was classified into the basic-type PR1, the major PR1 type. The basictype PR1 (pl at 8 10) significantly enhance antimicrobial and stress resistance (Li et al., 2011) and play a role in signal transition of host defense response (van Loon and Van Strien, 1999; van Loon et al., 2006). PsPR1 sequence showed homology to the sequences from the other plants and belonged to the basic-type of PR1, which may explain its antifungal activity and critical role in the poplar defense response. The Gns proteins with pl 6.2 and 8.2 were encoded by multiple-genes, and were involved in resistance and development (Kini et al., 2000). The Gns gene sequence was conserved between PsGns and Gns from other species, which suggests its similar role in hydrolyzing the fungal cell wall and eliciting the defense response (Veluthakkal and Dasgupta, 2010). Therefore, PsGns with pl 7.32 was expected to be involved in the host resistance to the 
fungus. $P$. trichocarpa are reported to contain 42 TLP genes with diverse expressions in leaves infected with Melampsora spp (Rinaldi et al., 2007; Miranda et al., 2007; Azaiez et al., 2009; Petre et al., 2011). The length and physicochemical properties of the deduced proteins of PsTLP1 (245) and PSTLP2 (225) were consistent with most of the P. trichocarpa TLPs ( 205-288 aa) (Zhao and Su, 2010; Petre et al., 2011). However, there were differences in the properties, such as pl, between PsTLP1 and PsTLP2 as well as different phylogenetic classification (in two groups). PsTLPs contain 16 conserved cysteine residues, similar to the large type of TLPs, compared with the small TLP group that contains only ten cysteine residues (Liu et al., 2010). According to the conserved domain analysis, PSTLP genes from $P$. szechuanica belong to the GH64-TLPSF superfamily. The conserved positions (cysteine), domains (TLP-PA) and motifs (REDDD) of PSTLPS may assist in predicting their function with regard to antifungal property, namely hydrolysis of $\beta-1,3$ glucan, which is usually found in fungal walls (Grenier et al., 1999; Petre et al., 2011). As $\mathrm{PAL}$ and $\mathrm{HAL}$ catalyze the conversion of L-phenylalanine to E-cinnamic acid and L-Histidine to E-Urocanic acid, respectively, the presence of PAL_HAL domain in PSPAL may suggest its role in the phenylpropanoid pathway (Baedeker and Schulz, 2002; Calabrese et al., 2004).

Previous studies reported that expression levels of some defense-related genes vary among different organs, and $D R$ genes were specifically expressed in certain organs of plants in response to biotic or abiotic stresses (Kao et al., 2002; Tachi et al., 2009). PsPR1, PsTLP1, PSTLP2, and PSPAL are observed to be up-regulated in SL, SP, SNL, and SB of $P$. szechuanica infected by MLP isolates $\mathrm{Sb}_{052}$ at $\sim$ 3-7 or $18 \mathrm{dpi}$ (Figure 6), suggesting that the expression of these genes was triggered not only in the inoculated leaves but also in the other organs of the infected poplar plants, and this induction might help to enhance the resistance of the whole plant to MLP.

As established from earlier reports, four of 12 PR1 genes were expressed at high levels in the roots with varying expression in the other organs of the healthy plant (Mitsuhara et al., 2008). Interestingly, we observed that the PSPR1 was also highly expressed in the roots than in the other organs of the uninfected $P$. szechuanica plant on $0 \mathrm{dpi}$, suggesting that the $P s P R 1$ is probably involved in root development (Figure 6). PsGns expression level in the organs of the uninfected P. szechuanica plant was similar to that of the major Gns genes, usually not expressed in the roots or stems and with lower level of expression in the leaves and buds of healthy plants (Veluthakkal and Dasgupta, 2010). PSPAL expression was abundant in the roots and top buds of the uninfected $P$. szechuanica plant, which was in conformance with results in Arabidopsis, and is possibly because the phenylpropanoid pathway is more active in the cells of roots than in other organs (Raes et al., 2003).

In this study, the expression levels of five PsDR (including PsPR1, PsGns, PsPAL, PsTLP1, and PsTLP2) genes in poplar leaves were markedly higher in the incompatible interaction than in the compatible interaction at most of the time points, indicating the PsPR genes were involved in the defense-related response. The PsDR genes expression levels peaked at $\sim 0.5-1$ or $2 \mathrm{dpi}$ in the leaves of incompatible interaction (Figure 7). It was observed that, during the infection, hyphae of MLP extended into the palisade mesophyll at $\sim 1-2 \mathrm{dpi}$, we deduced that the high expression of $P S P R$ at approximately the same time might be related to the basic defense, which resulted in the significant difference in the fungal biomass between the compatible and incompatible interactions (Rinaldi et al., 2007: Hacquard et al., 2011). In the incompatible interaction, programmed cell death of the host cells, accumulation of monolignols, and hypersensitive response (HR) around infection sites were observed after 1-2 dpi, accompanied by strong plant defense reaction (Rinaldi et al., 2007; Azaiez et al., 2009). In our study, the second peak of PsPR expression was observed at 4 or $7 \mathrm{dpi}$ in the leaves of plants with the incompatible interaction, which may indicate strong defense 
response at $\sim 4-7$ dpi. Although the functions of PR1 genes are not fully understood, some PR1 proteins were reported to possess antifungal activity against pathogens such as Phytophthora infestans (Alexander et al., 1993). Moreover, antifungal mechanisms of Gns and TLP genes were reported to be membrane permeabilization, $\beta$-glucan binding, and degradation, and inhibition of enzymes such as xylanases, $\alpha$-amylase, or trypsin (Min et al., 2004; Fierens et al., 2009). Poplar PAL was encoded by multiple genes with diverse sequences and expression patterns in response to biotic and abiotic stresses (Hamberger et al., 2007; Vanholme et al., 2010). In this study, PSPAL expression level was dramatically up-regulated from 1 to $2 \mathrm{dpi}$, and peaked at $2 \mathrm{dpi}$ in SL, which was concordant with increase of PAL activity at $2 \mathrm{dpi}$ in P. szehucanica in response to MLP (Chen et al., 2010). According to earlier reports, several genes of the phenylpropanoid pathways were induced at $2 \mathrm{dpi}$ in inoculated leaves, and monolignols were strongly accumulated at $2 \mathrm{dpi}$ in the incompatible interaction (Rinaldi et al., 2007). Thus, the PSPAL up-regulation in SL was considered to be associated with the deposition of lignin that strengthen the cell wall, and induction of secondary metabolic products that directly impeded the fungal growth or even killed the fungus in the poplar leaves. Therefore, the highly expression of these PsDR genes in the leaves of plants having incompatible interactions indicated that the PsDRs possess potential antifungal functions in the defense response to the rust fungus MLP.

\section{ACKNOWLEDGMENTS}

Research supported by the National Natural Science Foundation of China (Grant\#30872027).

\section{REFERENCES}

Alexander D, Goodman RM, Gut-Rella M, Glascock C, et al. (1993). Increased tolerance to two oomycete pathogens in transgenic tobacco expressing pathogenesis-related protein 1a. Proc. Natl. Acad. Sci. USA 90: 7327-7331. http://dx.doi. org/10.1073/pnas.90.15.7327

Azaiez A, Boyle B, Levée V and Séguin A (2009). Transcriptome profiling in hybrid poplar following interactions with Melampsora rust fungi. Mol. Plant Microbe Interact. 22: 190-200. http://dx.doi.org/10.1094/MPMI-22-2-0190

Baedeker M and Schulz GE (2002). Structures of two histidine ammonia-lyase modifications and implications for the catalytic mechanism. Eur. J. Biochem. 269: 1790-1797. http://dx.doi.org/10.1046/j.1432-1327.2002.02827.x

Bagal UR, Leebens-Mack JH, Lorenz WW and Dean JF (2012). The phenylalanine ammonia lyase (PAL) gene family shows a gymnosperm-specific lineage. BMC Genomics 13 (Suppl 3): S1.

Calabrese JC, Jordan DB, Boodhoo A, Sariaslani S, et al. (2004). Crystal structure of phenylalanine ammonia lyase: multiple helix dipoles implicated in catalysis. Biochemistry 43: 11403-11416. http://dx.doi.org/10.1021/bi049053+

Chen ZJ, Cao ZM, Gou XQ and Jiang ZR (2010). Dynamic Changes of Active Oxygen and Related Enzymes of the Host in Interaction between the Poplar and Melampsora larici-populina. Sci. Silvae Sin. 46: 101-106.

Chen ZJ, Cao ZM and Yu ZD (2014). Identification and characterization of differentially expressed genes during incompatible interaction between the foliar rust Melampsora larici-populina and poplar. Genet. Mol. Res. 13: 2082-2093. http://dx.doi. org/10.4238/2014.March.24.12

Covarelli L, Beccari G, Tosi L, Fabre B, et al. (2013). Three-year investigations on leaf rust of poplar cultivated for biomass production in Umbria, Central Italy. Biomass Bioenergy 49: 315-322. http://dx.doi.org/10.1016/j.biombioe.2012.12.032

Duplessis S, Major I, Martin F and Séguin A (2009). Poplar and pathogen interactions: insights from populus genome- wide analyses of resistance and defense gene families and gene expression profiling. Crit. Rev. Plant Sci. 28: 309-334. http:// dx.doi.org/10.1080/07352680903241063

Fernández C, Szyperski T, Bruyère T, Ramage P, et al. (1997). NMR solution structure of the pathogenesis-related protein P14a. J. Mol. Biol. 266: 576-593. http://dx.doi.org/10.1006/jmbi.1996.0772

Fierens E, Gebruers K, Voet AR, De Maeyer M, et al. (2009). Biochemical and structural characterization of TLXI, the Triticum aestivum L. thaumatin-like xylanase inhibitor. J. Enzyme Inhib. Med. Chem. 24: 646-654. http://dx.doi. org/10.1080/14756360802321831 


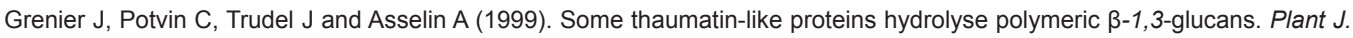
19: 473-480. http://dx.doi.org/10.1046/j.1365-313X.1999.00551.x

Hacquard S, Petre B, Frey P, Hecker A, et al. (2011). The poplar-poplar rust interaction: insights from genomics and transcriptomics. J. Pathog. Doi:10.4061/2011/716041.

Hamberger B, Ellis M, Friedmann M, Souza CA, et al. (2007). Genome-wide analyses of phenylpropanoid-related genes in Populus trichocarpa, Arabidopsis thaliana, and Oryza sativa: the Populus lignin toolbox and conservation and diversification of angiosperm gene families. Can. J. Bot. 85: 1182-1201. http://dx.doi.org/10.1139/B07-098

Kao YY, Harding SA and Tsai CJ (2002). Differential expression of two distinct phenylalanine ammonia-lyase genes in condensed tannin-accumulating and lignifying cells of quaking aspen. Plant Physiol. 130: 796-807. http://dx.doi. org/10.1104/pp.006262

Kini KR, Vasanthi NS and Shetty HS (2000). Induction of $\beta-1,3-$ glucanase in seedlings of pearl millet in response to infection by Sclerospora graminicola. Eur. J. Plant Pathol. 106: 267-274. http://dx.doi.org/10.1023/A:1008771124782

Klarzynski O, Plesse B, Joubert JM, Yvin JC, et al. (2000). Linear $\beta-1,3$ glucans are elicitors of defense responses in tobacco. Plant Physiol. 124: 1027-1038. http://dx.doi.org/10.1104/pp.124.3.1027

Li ZT, Dhekney SA and Gray DJ (2011). PR-1 gene family of grapevine: a uniquely duplicated PR-1 gene from a Vitis interspecific hybrid confers high level resistance to bacterial disease in transgenic tobacco. Plant Cell Rep. 30: 1-11. http://dx.doi. org/10.1007/s00299-010-0934-5

Liu JJ, Sturrock R and Ekramoddoullah AK (2010). The superfamily of thaumatin-like proteins: its origin, evolution, and expression towards biological function. Plant Cell Rep. 29: 419-436. http://dx.doi.org/10.1007/s00299-010-0826-8

Livak KJ and Schmittgen TD (2001). Analysis of relative gene expression data using real-time quantitative PCR and the 2(-Delta Delta C(T)) Method. Methods 25: 402-408. http://dx.doi.org/10.1006/meth.2001.1262

Min K, Ha SC, Hasegawa PM, Bressan RA, et al. (2004). Crystal structure of osmotin, a plant antifungal protein. Proteins 54: 170-173. http://dx.doi.org/10.1002/prot.10571

Miranda M, Ralph SG, Mellway R, White R, et al. (2007). The transcriptional response of hybrid poplar (Populus trichocarpa x $P$. deltoides) to infection by Melampsora medusae leaf rust involves induction of flavonoid pathway genes leading to the accumulation of proanthocyanidins. Mol. Plant Microbe Interact. 20: 816-831 http://dx.doi.org/10.1094/MPMI-20-7-0816.

Mitsuhara I, Iwai T, Seo S, Yanagawa Y, et al. (2008). Characteristic expression of twelve rice PR1 family genes in response to pathogen infection, wounding, and defense-related signal compounds (121/180). Mol. Genet. Genomics 279: 415-427. http://dx.doi.org/10.1007/s00438-008-0322-9

Petre B, Major I, Rouhier N and Duplessis S (2011). Genome-wide analysis of eukaryote thaumatin-like proteins (TLPs) with an emphasis on poplar. BMC Plant Biol. 11: 33. http://dx.doi.org/10.1186/1471-2229-11-33

Pinon J and Frey P (2005). Interactions between poplar clones and Melampsora populations and their implications for breeding for durable resistance. In: Rust Diseases of Willow and Poplar (Pei MH and McCracken AR, eds.). CAB International, Wallingford, 139-154.

Raes J, Rohde A, Christensen JH, Van de Peer Y, et al. (2003). Genome-wide characterization of the lignification toolbox in Arabidopsis. Plant Physiol. 133: 1051-1071. http://dx.doi.org/10.1104/pp.103.026484

Rinaldi C, Kohler A, Frey P, Duchaussoy F, et al. (2007). Transcript profiling of poplar leaves upon infection with compatible and incompatible strains of the foliar rust Melampsora larici-populina. Plant Physiol. 144: 347-366. http://dx.doi.org/10.1104/ pp.106.094987

Shatters RGJ, Jr., Boykin LM, Lapointe SL, Hunter WB, et al. (2006). Phylogenetic and structural relationships of the PR5 gene family reveal an ancient multigene family conserved in plants and select animal taxa. J. Mol. Evol. 63: 12-29 http://dx.doi. org/10.1007/s00239-005-0053-z.

Tachi H, Fukuda-Yamada K, Kojima T, Shiraiwa M, et al. (2009). Molecular characterization of a novel soybean gene encoding a neutral PR-5 protein induced by high-salt stress. Plant Physiol. Biochem. 47: 73-79. http://dx.doi.org/10.1016/j. plaphy.2008.09.012

van Loon LC and Van Strien EA (1999). The families of pathogenesis-related proteins, their activities, and comparative analysis of PR-1 type proteins. Physiol. Mol. Plant Pathol. 55: 85-97. http://dx.doi.org/10.1006/pmpp.1999.0213

van Loon LC, Rep M and Pieterse CM (2006). Significance of inducible defense-related proteins in infected plants. Annu. Rev. Phytopathol. 44: 135-162 http://dx.doi.org/10.1146/annurev.phyto.44.070505.143425.

Vanholme R, Demedts B, Morreel K, Ralph J, et al. (2010). Lignin biosynthesis and structure. Plant Physiol. 153: 895-905. http://dx.doi.org/10.1104/pp.110.155119

Veluthakkal R and Dasgupta MG (2010). Pathogenesis-related genes and proteins in forest tree species. Trees (Berl.) 24: 9931006. http://dx.doi.org/10.1007/s00468-010-0489-7

Wan ZB, Li Y, Chen Y, Zhang X, et al. (2013). Melampsora larici-populina, the main rust pathogen, causes loss in biomass production of black cottonwood plantations in the south of China. Phytoparasitica 41: 337-344. http://dx.doi.org/10.1007/ 
$\underline{\text { s12600-013-0294-0 }}$

Wang X, Tang C, Deng L, Cai G, et al. (2010). Characterization of a pathogenesis-related thaumatin-like protein gene TaPR5 from wheat induced by stripe rust fungus. Physiol. Plant. 139: 27-38. http://dx.doi.org/10.1111/j.1399-3054.2009.01338.x Zhao JP and Su XH (2010). Patterns of molecular evolution and predicted function in thaumatin-like proteins of Populus trichocarpa. Planta 232: 949-962. http://dx.doi.org/10.1007/s00425-010-1218-6

\section{Supplementary material}

Figure S1. Conserved domain analysis of five PsDR genes.

Figure S2. Phylogenetic tree of PsPR1 with PR1 from various plant species. The sequence name and GenBank accession Nos. are the same as in Figure 2.

Figure S3. Phylogenetic tree of PsGns with Gns from various plant species. The first two letters correspond to the initial letters of the genus and species names of the organisms. Sequence name and GenBank accession Nos. are as follows: Populus trichocarpa, PtGns (XP_002299791.2); Populus tremula x Populus tremuloides, PtrGns (ADW08742.1); Morus notabilis, MnGns (XP_010101247.1); Fragaria $x$ ananassa, FaGns (AAX81590.1); Pyrus pyrifolia, PpGns (AFP23132.1); Musa AB Group, MuGns (AIT56224.1); Hevea brasiliensis, HbGns (AAP87281.1); Vitis riparia, VrGns (ACD45060.1).

Figure S4. Phylogenetic tree of PsPAL with PAL from various plant species. The sequence name and GenBank accession Nos. are the same as in Figure 4.

http://www.geneticsmr.com/year2016/vol15-1/pdf/gmr7314_supplementary.pdf 